\title{
A Report of Two Cases of Adventitial Cystic Disease of the Popliteal Artery
}

\author{
Doo Jae Lee, $\mathrm{MD}^{1}$, Hyun Oh Park, $\mathrm{MD}^{2}$, Ha Nee Jang, $\mathrm{MD}^{3}$, Ki Nyun Kim, $\mathrm{MD}^{2}$, Jun Ho Yang, MD \\ Seong Ho Moon, $\mathrm{MD}^{2}$, Joung Hun Byun, $\mathrm{MD}^{2}$, Sung Hwan Kim, $\mathrm{MD}^{2}$, Jun Young Choi, $\mathrm{MD}^{4}$, \\ In Seok Jang, $\mathrm{MD}^{4}$, Jong Woo Kim, $\mathrm{MD}^{2}$, and Chung Eun Lee, $\mathrm{MD}^{2}$ \\ ${ }^{1}$ Division of Foot and Ankle, Department of Orthopedic Surgery, Seoul National University Hospital, Seoul; ${ }^{2}$ Department of Thoracic and Cardiovascular Surgery, \\ Gyeongsang National University Changwon Hospital, Gyeongsang National University College of Medicine, Changwon; ${ }^{3}$ Department of Internal Medicine, Gyeongsang \\ National University Hospital, Gyeongsang National University College of Medicine, Jinju; ${ }^{4}$ Department of Thoracic and Cardiovascular Surgery, Gyeongsang National \\ University Hospital, Gyeongsang National University College of Medicine, Jinju, Korea
}

\begin{abstract}
Two patients were admitted to our department because of recent aggravation of claudication in the leg, which was exacerbated by walking. They were diagnosed as having a Baker cyst or acute thrombosis in the popliteal fossa at another hospital. There was no evidence of ischemia, and the ankle brachial index was normal. Computed tomography and magnetic resonance imaging were performed, revealing a cystic mass of the popliteal artery (PA). Intraoperatively, the cystic lesion was found within the adventitia of the PA; based on the biopsy findings, both patients were diagnosed as having adventitial cystic disease of the PA.
\end{abstract}

Keywords: Popliteal artery, Adventitia, Cyst

Adventitial cystic disease (ACD) is a rare disease that occurs when adventitial mucoid cysts develop inside of the artery and vein, causing narrowing of the vessel lumen ${ }^{1)}$. Patients are usually middle age and do not have risk factors of atherosclerosis ${ }^{2,3)}$. ACD is a rare disease that mainly affects the popliteal artery (PA) and usually manifests as intermittent claudication during exercise $^{3)}$. ACD can be often misdiagnosed as atherosclerosis, an aneurysm, traumatic injury, a Backer cyst, or an arterial embolus, and a preoperative examination is important for making an accurate diagnosis ${ }^{4}$.

Received July 28, 2017; Revised (1st) August 18, 2017;

(2nd) September 22, 2017; (3rd) September 27, 2017;

(4th) October 6, 2017; Accepted October 7, 2017

Correspondence to: Chung Eun Lee, MD

Department of Thoracic and Cardiovascular Surgery, Institute of Health Science, Gyeongsang National University Hospital, 79 Gangnam-ro, Jinju 52727, Korea

Tel: +82-55-750-8124, Fax: +82-55-753-8138

E-mail: ilikerem@hanmail.net

This is an Open Access article distributed under the terms of the Creative Commons Attribution Non-Commercial License (http://creativecommons.org/licenses/by-nc/4.0/) which permits unrestricted non-commercial use, distribution, and reproduction in any medium, provided the original work is properly cited.
There have been several reports of ACD of PA. Previous reports have mainly described the pathogenesis, clinical features, and management of $\mathrm{ACD}^{5,6)}$ or imaging findings ${ }^{7,8)}$. Herein, we describe the processes of treating ACD of the PA and demonstrate the importance of an accurate preoperative examination. The first patient was diagnosed as having ACD based on an accurate preoperative examination. However, the second patient was misdiagnosed as having a thrombotic occlusion of the PA due to an incomplete preoperative examination but was later diagnosed as having ACD postoperatively.

\section{Case Reports}

\section{Case 1}

A 33-year-old man was referred to our institution because of edema and pain in his left lower extremity that had begun 8 days earlier. His risk factors for atherosclerotic diseases included smoking (1 pack per day) but nothing else remarkable. Based on the results of the patient's ultrasonography done in another hospital, he was diagnosed as having acute thrombosis in the distal portion of the superficial femoral artery or a Baker cyst in the popliteal fossa. Computed tomography (CT) angiography was 
performed to determine the site and extent of stenosis and evaluate the entire circulation system, and we found a cystic lesion with mild peripheral enhancement of the popliteal vessels. There was significant focal eccentric narrowing of the PA (Fig. 1). The possibility of ACD of the PA was high, but it was not completely distinguished from ACD originating from the popliteal vein. Hence, there was a need to determine if it was either ACD originating from the popliteal vein or PA, so we performed magnetic resonance imaging (MRI). Cystic lesions close to both $\mathrm{P}$ walls were seen as areas of low to intermediate signal intensity on T1weighted MRI (Fig. 2A) and as areas of high signal intensity on T2-weighted MRI (Fig. 2B). On the basis of characteristic imaging findings, we diagnosed him as having a cyst originating from the PA. Intraoperatively, we identified a cyst originating from the PA. We performed cyst evacuation and finished the operation after confirming that blood flow to the PA had been recovered. On the basis of the biopsy result, we made a final diagnosis of ACD. Periodic follow-up studies showed no local recurrence until 32 months postoperatively.

\section{Case 2}

A 66-year-old man visited our institution with the chief complaint of pain and claudication in the right leg that had begun 6 months previously. He was diagnosed as having a Baker cyst or thrombotic occlusion of the PA at another hospital. CT angiography was performed, and we diagnosed him as thrombotic occlusion of the PA (Fig. 3A and B). We planned and performed an operation to remove the thrombus of the PA. However, we identified a cystic mass originating from the PA unlike the preoperative diagnosis (Fig. 4). The diameter of the PA was still narrow after removing the lesion, so we performed graft interposition using a 6-mm polytetrafluoroethylene graft (Terumo Cardiovascular

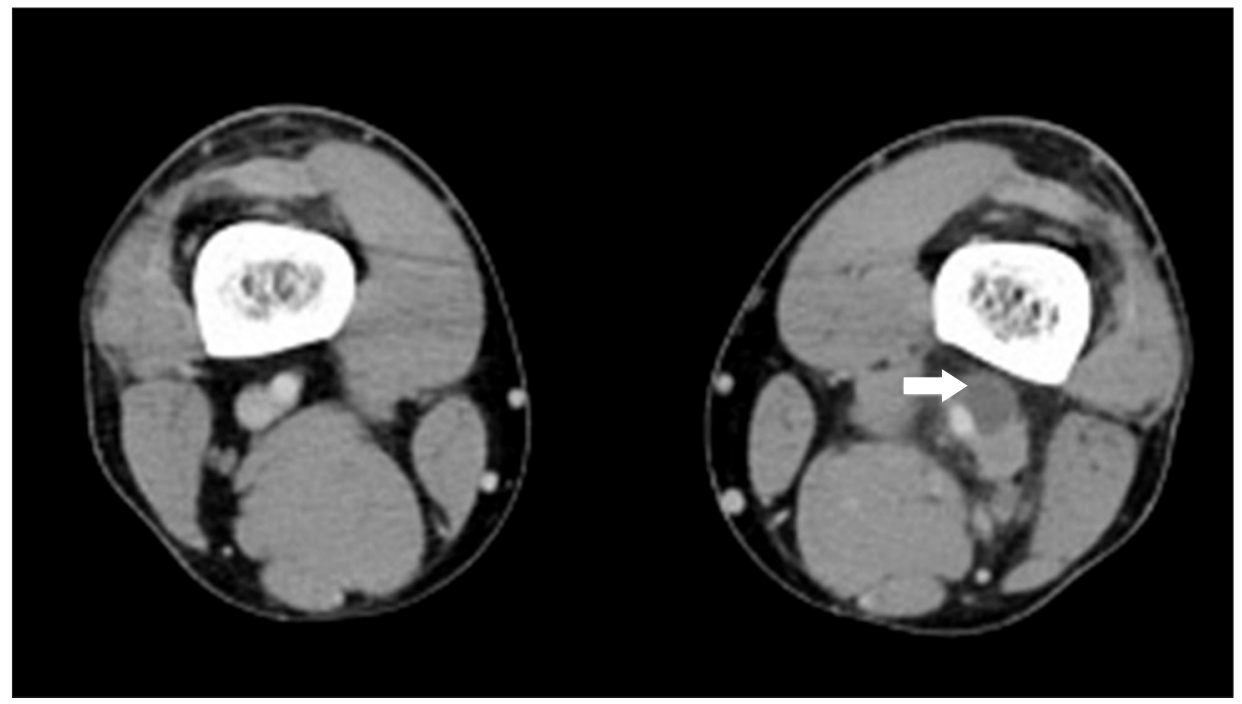

Fig. 1. Case 1: A 33-year-old man. Computed tomography angiogram showing a cystic lesion (white arrow) causing marked eccentric narrowing of the popliteal artery.
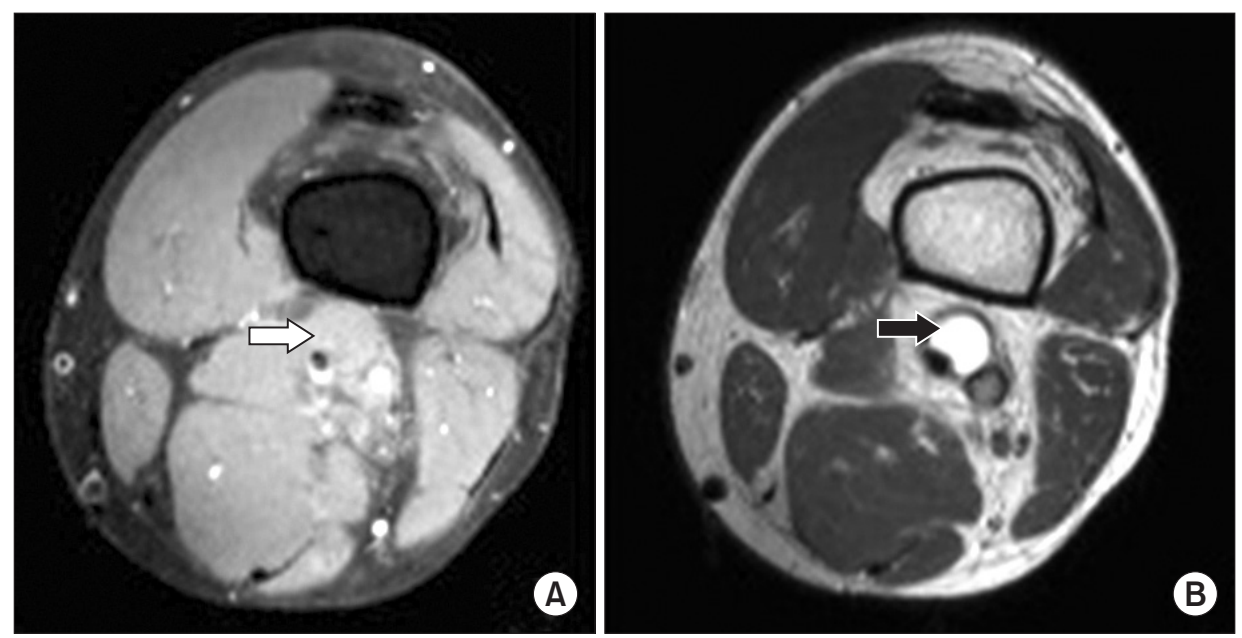

Fig. 2. Case 1: A 33-year-old man. (A) T1weighted axial magnetic resonance imaging (MRI) scan of the knee showing a cystic lesion (white arrow) with intermediate signal intensity due to the mucin content surrounding the popliteal artery. (B) T2weighted axial MRI scan of the left knee showing a high signal intensity cystic lesion (black arrow) of the popliteal artery. 

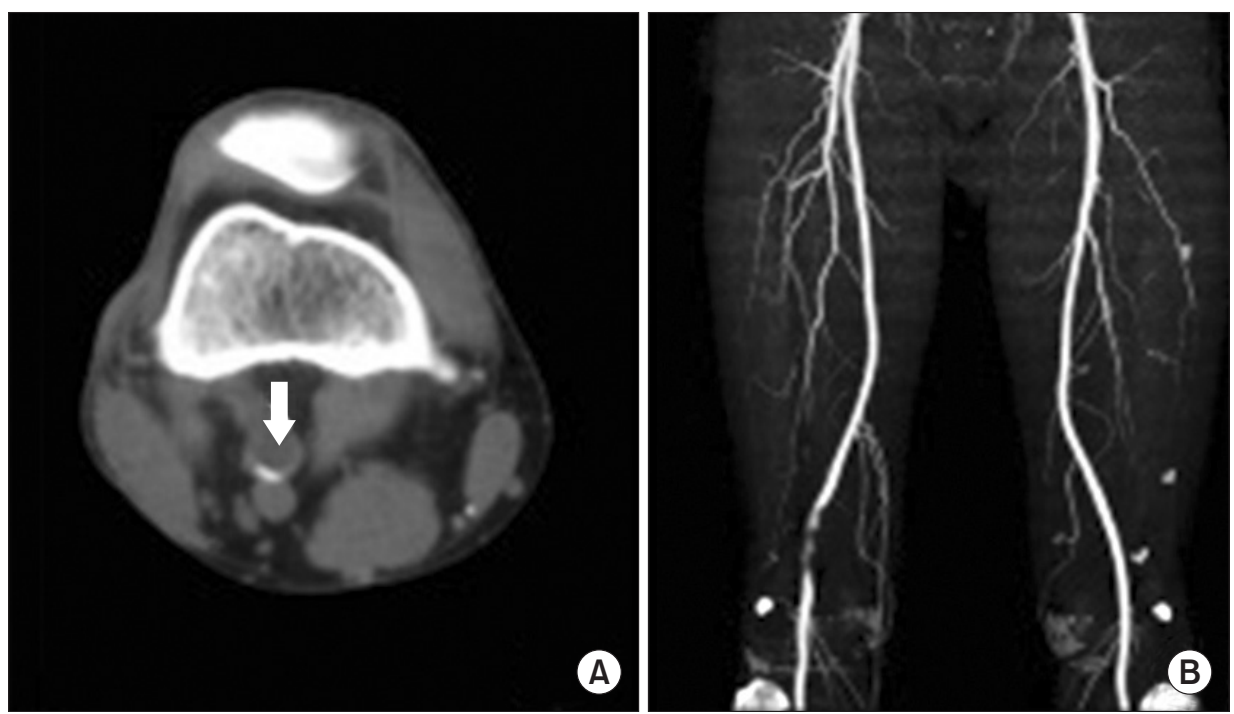

Fig. 3. Case 2: A 66-year-old man. (A) Axial computed tomography (CT) angiogram showing a cystic lesion of the popliteal artery. Cystic lesions are large and eccentric, and they may displace the artery to one side-the so-called scimitar sign (white arrow). (B) Three-dimensional CT reconstruction image showing partial occlusion of the popliteal artery.

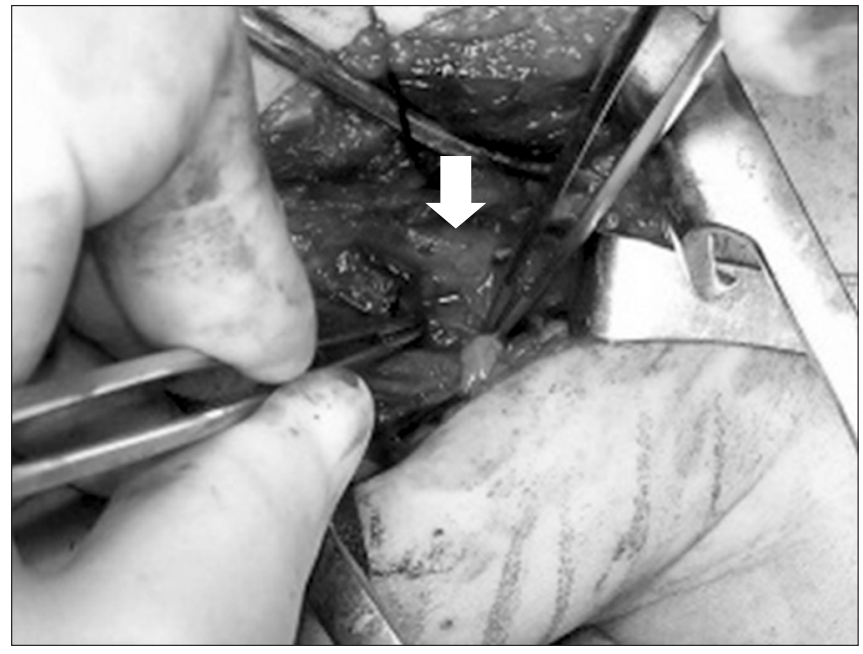

Fig. 4. Case 2: A 66-year-old man. Intraoperative finding of the adventitial cyst (white arrow) in the surrounding part of the popliteal artery.

Systems Corp., Ann Arbor, MI, USA). Postoperatively, we found that the blood flow to the PA was completely recovered. Later, we made a final diagnosis of ACD based on the biopsy finding (Fig. 5). Periodic follow-up studies showed no local recurrence until 35 months postoperatively.

\section{Discussion}

ACD is a non-atherosclerotic vascular disease that causes local stenosis or occlusion of the peripheral vessels. Shortly after Atkins and $\mathrm{Key}^{1)}$ first identified a case of ACD in the right external iliac artery in 1947, Ejrup and Hiertonn ${ }^{2)}$ reported a case of ACD of the PA for the first time in 1954. ACD of the PA is found incidentally in 1 of 1,200 patients complaining of claudication

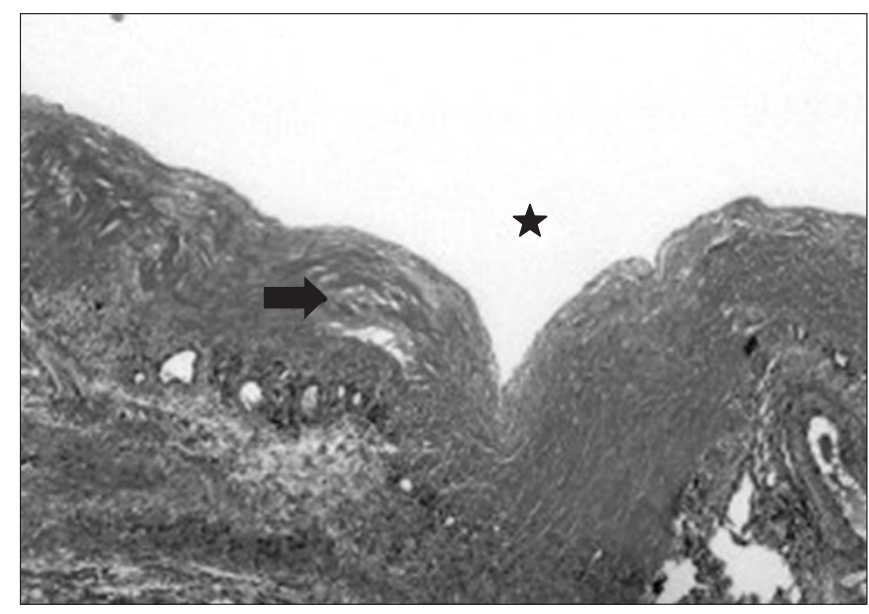

Fig. 5. Case 2: a 66-year-old man. Photomicrograph showing the cystic space (asterisk) filled with gelatinous material and the dissected arterial wall (arrow) with collagen bundles via mucoid degeneration (H\&E, $\times 20$ ).

of their lower extremity or in 1 of 1,000 patients who undergo angiography. It is about 15 times more common in men than in women $^{3)}$. The average age of diagnosis is the fourth decade of life for men and fifth decade of life for women ${ }^{3,5)}$.

This disease should be suspected and an imaging examination should be performed when a person with a relatively low risk of developing cardiovascular diseases experiences sudden claudication unilaterally ${ }^{4,6)}$. Ultrasonography with color Doppler shows characteristic features of an anechoic smooth-walled mass seen in the wall of the PA. It shows good posterior enhancement and no flow within the cysts ${ }^{7}$. CT angiography may demonstrate narrowing of the vessels with an absence of collateral vessels. When cystic lesions are large and eccentric, they may displace the artery 
to one side-the so-called scimitar sign ${ }^{4)}$. The finding of MRI varies depending on the distribution and size of the cyst. T1weighted MRI shows that individual lesions have a variable signal dependent on the mucoid content, and T2-weighted MRI shows that individual lesions have a high signal intensity, confirming the diagnosis ${ }^{8)}$. When the lesions are large, MRI scans can also show the scimitar sign ${ }^{4}$.

We described two patients with ACD. The first patient was diagnosed as having ACD with an accurate preoperative examination, but the second patient was misdiagnosed as having thrombotic occlusion of the PA. ACD can be misdiagnosed as atherosclerosis, aneurysm, traumatic injury, a Baker cyst, or arterial embolus. Therefore, ACD should be included in the differential diagnosis when a patient with a relatively low risk of cardiovascular disease experiences sudden claudication, and physicians should perform various examinations to make an accurate diagnosis.

\section{Conflict of Interest}

No potential conflict of interest relevant to this article was reported.

\section{References}

1. Atkins HJ, Key JA. A case of myxomatous tumour arising in the adventitia of the left external iliac artery; case report. Br J Surg. 1947;34:426.

2. Ejrup B, Hiertonn T. Intermittent claudication; three cases treated by free vein graft. Acta Chir Scand. 1954;108:217-30.

3. Flanigan DP, Burnham SJ, Goodreau JJ, Bergan JJ. Summary of cases of adventitial cystic disease of the popliteal artery. Ann Surg. 1979;189:165-75.

4. Wright LB, Matchett WJ, Cruz CP, James CA, Culp WC, Eidt JF, McCowan TC. Popliteal artery disease: diagnosis and treatment. Radiographics. 2004;24:467-79.

5. Zhang H, Zhang Y, Wang Q, Zhao WG, Wang JJ. Cystic adventitial disease of the popliteal artery: report of two cases. Surg Today. 2014;44:1760-3.

6. Kwon DJ, Lee WY, Kim KI, Min SK, Park SW, Kim YC, Lee $\mathrm{KB}$. Cystic adventitial disease of the popliteal artery: a case report. J Korean Knee Soc. 2005;17:258-61.

7. Franca M, Pinto J, Machado R, Fernandez GC. Case 157: bilateral adventitial cystic disease of the popliteal artery. Radiology. 2010;255:655-60.

8. Peterson JJ, Kransdorf MJ, Bancroft LW, Murphey MD. Imaging characteristics of cystic adventitial disease of the peripheral arteries: presentation as soft-tissue masses. AJR Am J Roentgenol. 2003;180:621-5. 